\title{
EXISTENCE AND UNIQUENESS OF CONSTRAINED GLOBALLY OPTIMAL FEEDBACK CONTROLS IN A LINEAR-QUADRATIC FRAMEWORK
}

\author{
YASHAN XU
}

Received 14 January 2006; Revised 5 March 2006; Accepted 15 March 2006

A constrained closed-loop optimal control problem is considered in a linear-quadratic framework. To solve the problem, a special type open-loop optimal control problem and a standard open-loop optimal control problem are introduced and carefully studied, via which the existence and uniqueness of the globally optimal closed-loop control is established by a synthesis method.

Copyright (c) 2006 Yashan Xu. This is an open access article distributed under the Creative Commons Attribution License, which permits unrestricted use, distribution, and reproduction in any medium, provided the original work is properly cited.

\section{Introduction}

Let us recall the classical linear-quadratic (LQ, for short) optimal control problem. Consider the following controlled linear state equation:

$$
\begin{gathered}
\dot{y}(s)=A(s) y(s)+B(s) u(s), \quad s \in[t, T], \\
y(t)=x,
\end{gathered}
$$

where $y(\cdot)$ is the state, valued in $\mathbb{R}^{n}$, with the initial state $x \in \mathbb{R}^{n}$ at the initial time $t \in$ $[0, T), u(\cdot)$ is the control taken from $L^{2}\left(t, T ; \mathbb{R}^{m}\right)$ (the space of all square integrable functions valued in $\mathbb{R}^{m}$ defined on $\left.[t, T]\right), A(\cdot) \in C\left([0, T] ; \mathbb{R}^{n \times n}\right)$, and $B(\cdot) \in C\left([0, T] ; \mathbb{R}^{n \times m}\right)$. The cost functional is defined to be

$$
J(t, x ; u(\cdot))=\int_{t}^{T}[\langle Q(s) y(s), y(s)\rangle+\langle R(s) u(s), u(s)\rangle] d s+\langle M y(T), y(T)\rangle,
$$

where $Q(\cdot)=Q(\cdot)^{\top} \in C\left([0, T] ; \mathbb{R}^{n \times n}\right), R(\cdot)=R(\cdot)^{\top} \in C\left([0, T] ; \mathbb{R}^{m \times m}\right)$, and $M=M^{\top} \in$ $\mathbb{R}^{n \times n}$. The classical LQ problem can be stated as follows. 
2 Existence and uniqueness of globally optimal feedback controls

Problem 1.1 (LQ). For any given $(t, x) \in[0, T) \times \mathbb{R}^{n}$, find a $\bar{u}^{t, x}(\cdot) \in L^{2}\left(t, T ; \mathbb{R}^{m}\right)$ such that

$$
J\left(t, x ; \bar{u}^{t, x}(\cdot)\right)=\inf _{u(\cdot) \in L^{2}\left(t, T ; \mathbb{R}^{m}\right)} J(t, x ; u(\cdot)) \equiv V(t, x) .
$$

Any $\bar{u}^{t, x}(\cdot) \in L^{2}\left(t, T ; \mathbb{R}^{m}\right)$ satisfying (1.3) is called an open-loop optimal control of Problem 1.1 at $(t, x)$. Function $V:[0, T] \times \mathbb{R}^{n} \rightarrow \mathbb{R}$ defined in (1.3) is called the open-loop value function of Problem 1.1. Note that, by definition,

$$
V(T, x)=\langle M x, x\rangle, \quad \forall x \in \mathbb{R}^{n} .
$$

We also emphasize that the open-loop optimal control $\bar{u}^{t, x}(\cdot)$ depends on the initial condition $(t, x)$.

Classical LQ theory is pretty mature $[1,11]$, and it has many applications in engineering as well as various other related fields $[1,4]$. Among many other things, the standard LQ theory says that under some mild conditions, say,

$$
Q(s) \geq 0, \quad R(s) \geq \delta I, \quad \forall s \in[0, T], M \geq 0,
$$

where $\delta>0$, for any $(t, x) \in[0, T) \times \mathbb{R}^{n}$, Problem 1.1 admits a unique open-loop optimal control $\bar{u}^{t, x}(\cdot)$ at $(t, x)$. Moreover, $\bar{u}^{t, x}(\cdot)$ admits a closed-loop representation, by which we mean that there exists a unique symmetric matrix-valued function $P(\cdot)$ satisfying a Riccati differential equation:

$$
\begin{gathered}
\dot{P}(s)+P(s) A(s)+A(s)^{\top} P(s)+Q(s)-P(s) B(s) R(s)^{-1} B(s)^{\top} P(s)=0, \quad s \in[0, T], \\
P(T)=M,
\end{gathered}
$$

such that

$$
\bar{u}^{t, x}(s)=-R(s)^{-1} B(s)^{\top} P(s) \bar{y}^{t, x}(s), \quad s \in[t, T]
$$

where $\bar{y}^{t, x}(\cdot)$ is the state trajectory corresponding to $\bar{u}^{t, x}(\cdot)$. Note that since $P(\cdot)$ is independent of the initial condition $(t, x)$, representation (1.7) is uniform in $(t, x)$.

Representation (1.7) suggests that we might directly consider the following state equation (compare with (1.1)):

$$
\begin{gathered}
\dot{y}(s)=A(s) y(s)+B(s) \varphi(s, y(s)), \quad s \in[t, T], \\
y(t)=x,
\end{gathered}
$$

with the cost functional

$$
J(t, x ; \varphi(\cdot, \cdot))=\int_{t}^{T}[\langle Q(s) y(s), y(s)\rangle+\langle R(s) \varphi(s, y(s)), \varphi(s, y(s))\rangle] d s+\langle M y(T), y(T)\rangle .
$$

In order for the state equation (1.8) to be well-posed, it is natural to require that $y \mapsto$ $\varphi(s, y)$ be uniformly Lipschitz. For convenience, let us denote this class of functions by $\Phi$. 
Then, for any $\varphi(\cdot, \cdot) \in \Phi$, and $(t, x) \in[0, T] \times \mathbb{R}^{n}$, the cost functional (1.9) will be well defined, and we can pose a new optimal control problem as follows.

Problem $1.2(\mathrm{LQ})^{F}$. Find a $\bar{\varphi}(\cdot, \cdot) \in \Phi$ such that

$$
J(t, x ; \bar{\varphi}(\cdot, \cdot))=\inf _{\varphi(\cdot, \cdot) \in \Phi} J(t, x ; \varphi(\cdot, \cdot)) \equiv \bar{V}(t, x), \quad \forall(t, x) \in[0, T] \times \mathbb{R}^{n},
$$

where and hereafter the superscript " $F$ " means feedback.

Any $\bar{\varphi}(\cdot, \cdot) \in \Phi$ satisfying $(1.10)$ is called an optimal control of Problem 1.2, or a closed-loop optimal control of Problem 1.1. Function $\bar{V}:[0, T] \times \mathbb{R}^{n} \rightarrow \mathbb{R}$ defined in (1.10) is called the closed-loop value function of Problem 1.1.

It turns out that under conditions (1.5) Problems 1.1 and 1.2 are essentially equivalent. To be more precise, we state the following result.

Proposition 1.3. Let conditions (1.5) hold. Then Riccati equation (1.6) admits a unique solution $P(\cdot)$ and, for any $(t, x) \in[0, T] \times \mathbb{R}^{n}$, Problem 1.1 admits a unique open-loop optimal control $\bar{u}^{t, x}(\cdot)$ at $(t, x)$, which admits a closed-loop representation (1.7). If

$$
\bar{\varphi}(s, y)=-R(s)^{-1} P(s) B(s)^{\top} y, \quad(s, y) \in[0, T] \times \mathbb{R}^{n},
$$

is defined, then $\bar{\varphi}(\cdot, \cdot) \in \Phi$ and it is an optimal control of Problem 1.2. Moreover,

$$
\bar{\varphi}(s, y)=\bar{u}^{s, y}(s), \quad \forall(s, y) \in[0, T] \times \mathbb{R}^{n} .
$$

Further, (1.11) is the only optimal control of Problem 1.2.

The above result roughly tells us that, for Problem 1.1, an open-loop optimal control admits a closed-loop representation, and it leads to a closed-loop optimal control. Conversely, any closed-loop optimal control must be a linear feedback control, and it leads to an open-loop optimal control.

The above seems to be a perfect story. However, in a number of interesting physical problems, it is necessary to impose some additional constraints upon the control and/or the state. Examples are control with piecewise constraints [14], and energy constraints [7], control with linear constraints [3], state constraints [8,9], and path constraints $[12,17]$, state and control with linear inequality constraints $[6,10,16]$ and quadratic constraints [13], and so forth. It should be pointed out that all the above-mentioned works only involved open-loop controls, for which one can apply Pontryagin's maximum principle. We have not seen works touched the constrained closed-loop control problems, to the author's best knowledge. This author has discussed LQ control problem with constrained state linear feedback [18]

$$
\varphi(s, y)=K(s) y, \quad\|K(s)\| \leq k,
$$

where $k$ is a given constant. In this paper, we consider the feedback controls $\varphi(\cdot, \cdot)$ that satisfy the following constraint:

$$
\|\varphi(s, y)\| \leq k\|y\|, \quad(s, y) \in[0, T] \times \mathbb{R}^{n},
$$

for some given constant $k>0$. Clearly this situation is a natural extension of (1.13). 


\section{Existence and uniqueness of globally optimal feedback controls}

To make the above type constraints more appealing, let us present an interesting example. Suppose that an aircraft flies in a convection layer which satisfies international atmosphere standard. The flight depends on its airscrew. Suppose that the biggest turn velocity of the airscrew is given, then the biggest impetus of the aircraft is a linear function of the air density of its position. If we let the control variable $u$ be the impetus of the aircraft, let variable $h$ be the height of the position of the aircraft, and let $\rho(h)$ be the air density depending on $h$, then the following constraint holds:

$$
|u(h)| \leq C \rho(h),
$$

where $C$ is a fixed constant. According to the international atmosphere standard, it follows that

$$
\rho(h)=\rho_{0}\left(1-\frac{a h}{T_{0}}\right)^{g / a R-1},
$$

where $\rho_{0}$ is the air density at the the sea lever, $T_{0}$ is the temperature at the the sea lever, $a$ is the descending rate of temperature, $g$ is the acceleration of gravity, and $R$ is a constant [15].

Now if we take $y=f(h)=\left(1-a h / T_{0}\right)^{g / a R-1}$ as the state variable, then $\hat{u}(y) \equiv u\left(f^{-1}(y)\right)$ satisfies

$$
|\hat{u}(y)| \leq C \rho_{0} y=C \rho_{0}|y|
$$

which has the form of (1.14).

The purpose of this paper is to study an optimal control problem for (1.8)-(1.9) with the constraint (1.14). Let us now make it precise.

For given $(k, t, x) \in(0,+\infty) \times[0, T) \times \mathbb{R}^{n}$, denote by $u_{k}^{F}(t, x)$ the set of all measurable functions $\varphi:[0, T] \times \mathbb{R}^{n} \rightarrow \mathbb{R}^{m}$ satisfying (1.14) and state equation (1.8) admits a unique strong solution for the given initial condition $(t, x)$. Further, let

$$
u_{k}^{F}=\bigcap_{(t, x) \in[0, T) \times \mathbb{R}^{n}} u_{k}^{F}(t, x) .
$$

Any $\varphi(\cdot, \cdot) \in u_{k}^{F}$ is called a feedback control. Note that $u_{k}^{F}$ contains all the functions $\varphi(\cdot, \cdot) \in \Phi$ (i.e., $y \mapsto \varphi(s, y)$ is uniformly Lipschitz) satisfying $\varphi(\cdot, 0)=0$. But, $\boldsymbol{u}_{k}^{F}$ may also contain functions that are not in $\Phi$. Here is an example.

Example 1.4. Let $n=m=1, A=0, B=1$, and $k=1$. Let

$$
\varphi(s, y)= \begin{cases}-y\left|\sin \left(\frac{1}{y}\right)\right|, & y \neq 0, \\ 0, & y=0,\end{cases}
$$

for all $s \in[0, T]$. Clearly, it is not uniformly Lipschitz on any neighborhood of $y=0$, for any given $s \in[0, T]$. Note that

$$
|\varphi(s, y)| \leq|y|=k|y|, \quad \forall(s, y) \in[0, T] \times \mathbb{R} .
$$


Thus, (1.14) holds with $k=1$. Further, state equation (1.8) under the above feedback control reads

$$
\begin{gathered}
\dot{y}(s)=\varphi(s, y(s)), \quad s \in[t, T], \\
y(t)=x .
\end{gathered}
$$

If $x>0$, any solution to the above equation fulfills

$$
y(s) \in\left[x e^{t-s}, x\right] \subseteq\left[x e^{t-T}, x\right] \quad \forall s \in[t, T] .
$$

Since $\varphi(s, \cdot)$ is Lipschitz on $\left[x^{t-T}, x\right]$, The solution of $(1.21)$ is unique. The case $x<0$ is similar. For $x=0$, any solution $y(\cdot)$ of $(1.21)$ satisfies

$$
\frac{d}{d s} y^{2}(s)=-2 y(s)^{2}\left|\sin \left(\frac{1}{y(s)}\right)\right| \chi_{\{y(s) \neq 0\}} \leq 0,
$$

leading to $y(\cdot)=0$, which is the unique solution of (1.21). Therefore $\varphi \in \boldsymbol{U}_{k}^{F}$.

From the above, we realize that the structure of $u_{k}^{F}$ is complicated. Hence, it is by no means obvious to check if a constructed feedback control, which might not be uniformly Lipschitz, belongs to $u_{k}^{F}$. Now, for any $\varphi(\cdot, \cdot) \in u_{k}^{F}$, the cost functional (1.9) is well defined. Hence we can pose the following constrained feedback control problem in our LQ framework.

Problem $1.5(\mathrm{LQ})_{k}^{F}(t, x)$. For the given $(t, x) \in[0, T] \times \mathbb{R}^{n}$, find a $\bar{\varphi}^{t, x}(\cdot, \cdot) \in \mathcal{U}_{k}^{F}(t, x)$ such that

$$
J\left(t, x ; \bar{\varphi}^{t, x}(\cdot, \cdot)\right)=\inf _{\varphi(\cdot, \cdot) \in \mathcal{U}_{k}^{F}(t, x)} J(t, x ; \varphi(\cdot, \cdot)) \equiv \bar{V}^{k}(t, x) .
$$

We refer to the above as a local constrained closed-loop LQ problem. The following is referred to as a global constrained closed-loop LQ problem.

Problem 1.6 (LQ $)_{k}^{F}$. Find a $\bar{\varphi}(\cdot, \cdot) \in \mathcal{U}_{k}^{F}$ such that $(1.24)$ holds for all $(t, x) \in[0, T] \times \mathbb{R}^{n}$.

We emphasize that $\bar{\varphi}^{t, x}(\cdot, \cdot)$ in Problem 1.5 might depend on $(t, x)$, whereas that in Problem 1.6 is required to be uniform in $(t, x) \in[0, T] \times \mathbb{R}^{n}$. Therefore, any $\bar{\varphi}^{t, x}(\cdot, \cdot) \in$ $U_{k}^{F}$ satisfying (1.24) is called an optimal control of Problem 1.5, or a locally optimal closedloop control of Problem 1.6, and if $\bar{\varphi}^{t, x}(\cdot, \cdot) \equiv \bar{\varphi}(\cdot, \cdot)$ satisfying $(1.24)$ for all $(t, x) \in[0, T] \times$ $\mathbb{R}^{n}$ is called a globally optimal closed-loop control of Problem 1.6, we call $\bar{V}^{k}(\cdot, \cdot)$ the value function of Problem 1.6.

We point out that due to the structure of $u_{k}^{F}$, Problem 1.6 is a nonstandard (openloop) optimal control problem. Hence, we do not expect to have the same HamiltonJacobi-Bellman (HJB, for short) equation for $\bar{V}^{k}(\cdot, \cdot)$ and Pontraygin-type maximum principle as a first-order necessary conditions for any optimal control $\bar{\varphi}(\cdot, \cdot)$. Further, even if we are in an LQ framework, in general, the value function $\bar{V}^{k}(\cdot, \cdot)$ might be nonquadratic with respect to the state variable (see Section 2 for an example). Thus, the Riccati equation technique (which is usually used for nonconstrained LQ problems) does not apply here either! All the above makes the currently problem interesting and challenging. 
6 Existence and uniqueness of globally optimal feedback controls

Our approach is to introduce a special type of open-loop optimal control problem via which we will obtain a kind of equivalence of our constrained closed-loop optimal control problem to a standard (open-loop) optimal control problem for which standard optimal control theory can apply.

The rest of this paper is organized as follows. In Section 2, we introduce an openloop optimal control with a special type of admissible controls. A relationship between such an open-loop control problem and the original closed-loop control problem is presented. Some properties of the corresponding value function will also be discussed. In Section 3, we introduce a standard open-loop optimal control problem which is essentially equivalent to the previous introduced open-loop control problem. Then the existence and uniqueness of the globally optimal closed-loop control is established by a synthesis method.

\section{Preliminary results}

In this section, we will introduce and discuss an open-loop constrained LQ problem which will be useful to study our constrained closed-loop control problem. In what follows, we fix a $k \in(0,+\infty)$.

First, for any given $(t, x) \in[0, T) \times \mathbb{R}^{n}$, let $u_{k}^{t, x}$ be the set of all open-loop controls $u(\cdot) \in L^{2}\left(t, T ; \mathbb{R}^{m}\right)$ such that

$$
\|u(s)\| \leq k\left\|y^{t, x}(s)\right\|, \quad \text { a.e. } s \in[t, T]
$$

with $y^{t, x}(\cdot)$ being the state trajectory of $(1.1)$ corresponding to $u(\cdot)$. If we let $\Psi(\cdot, \cdot)$ be the fundamental solution of $A(\cdot)$, that is,

$$
\frac{d}{d t} \Psi(t, s)=A(t) \Psi(t, s), \quad \Psi(s, s)=I,
$$

then

$$
y^{t, x}(s)=\Psi(s, t) x+\int_{t}^{s} \Psi(s, \tau) B(\tau) u(\tau) d \tau, \quad \forall s \in[t, T]
$$

Thus, (2.1) is equivalent to

$$
\|u(s)\| \leq k\left\|\Psi(s, t) x+\int_{t}^{s} \Psi(s, \tau) B(\tau) u(\tau) d \tau\right\|, \quad \text { a.e. } s \in[t, T] .
$$

We see that this constraint is nonconvex. Thus, the set $u_{k}^{t, x}$ is nonconvex. Consequently, $U_{k}^{t, x}$ must be a proper subset of $L^{2}\left(t, T ; \mathbb{R}^{m}\right)$. On the other hand, we clearly have the closedness of $u_{k}^{t, x}$ under the norm of $L^{2}\left(t, T ; \mathbb{R}^{m}\right)$, and, by Gronwall's inequality, $u_{k}^{t, x}$ is bounded. The following result is more interesting and will be useful.

Proposition 2.1. Let $(t, x) \in[0, T) \times \mathbb{R}^{n}$ and $u(\cdot) \in u_{k}^{t, x}$. Let $y^{t, x}(\cdot)$ be the corresponding state trajectory. Then the following are equivalent. 
(i) For any $s \in[t, T]$,

$$
y^{t, x}(s)=0
$$

(ii) For some $s \in[t, T],(2.5)$ holds.

(iii) $x=0$.

Proof. Let $\Psi(\cdot, \cdot)$ be the fundamental solution of $A(\cdot)$. Then

$$
y^{t, x}(r)=\Psi(r, s) y^{t, x}(s)+\int_{s}^{r} \Psi(r, \tau) B(\tau) u(\tau) d \tau, \quad \forall r, s \in[t, T] .
$$

Thus,

$$
\left\|y^{t, x}(r)\right\| \leq C\left\|y^{t, x}(s)\right\|+C k \int_{s}^{r}\|B(\tau)\|\left\|y^{t, x}(\tau)\right\| d \tau
$$

hereafter $C$ will represent an absolute constant, which can be different from line to line. Hence, by Gronwall's inequality, we have

$$
\left\|y^{t, x}(r)\right\| \leq C\left\|y^{t, x}(s)\right\|, \quad \forall r, s \in[t, T] .
$$

Then the equivalence of (i)-(iii) is obvious.

We now introduce the following definition.

Definition 2.2. Given an initial condition $(t, x) \in[0, T) \times \mathbb{R}^{n}$ and a feedback control $\varphi \in$ $u_{k}^{F}$,

(i) an open-loop control defined by

$$
u(\cdot)=\varphi\left(\cdot, y^{t, x}(\cdot)\right), \quad s \in[t, T],
$$

is called an open-loop control associated with the feedback control $\varphi(\cdot, \cdot) \in \boldsymbol{U}_{k}^{F}$ at $(t, x)$;

(ii) a set $\mathscr{A} \subseteq \mathcal{U}_{k}^{t, x}$ is called the open-loop control set associated with the feedback control set $\mathscr{\mathscr { B }} \subseteq \mathcal{U}_{k}^{F}$ at $(t, x)$ if $\mathscr{A}$ consists of all open-loop controls that associated with some feedback controls in $\mathscr{B}$ at $(t, x)$; denote $U_{k}^{F}(t, x)$ to be the open-loop control set associated with $u_{k}^{F}$ at $(t, x)$.

We point out that the map $\varphi(\cdot, \cdot) \mapsto u(\cdot)$ in Definition 2.2(i) is not necessarily oneto-one. In another word, different feedback controls might lead to the same open-loop control. Similarly, a set $\mathscr{A} \subseteq \mathcal{U}_{k}^{t, x}$ might be associated with more than one $\mathscr{S} \subseteq \mathcal{U}_{k}^{F}$ at $(t, x)$. The following result gives the relation between $u_{k}^{F}(t, x)$ and $u_{k}^{t, x}$.

Lemma 2.3. Let $(t, x) \in[0, T) \times \mathbb{R}^{n}$. Then $\boldsymbol{u}_{k}^{t, x}$ coincides with $\boldsymbol{u}_{k}^{F}(t, x)$.

Proof. According to the above definition, for any $\varphi(\cdot, \cdot) \in \mathcal{U}_{k}^{F}$, the open-loop control $u(\cdot)$ associated with $\varphi(\cdot, \cdot)$ belongs to $u_{k}^{t, x}$. This shows that $u_{k}^{F}(t, x) \subseteq u_{k}^{t, x}$. On the other hand, for any open-loop control $\hat{u}(\cdot) \in \mathcal{U}_{k}^{t, x}$, we would like to find a feedback control $\varphi(\cdot, \cdot)$ such that $\hat{u}(\cdot)$ is an open-loop control associated with it. In fact, by Proposition 2.1, 
8 Existence and uniqueness of globally optimal feedback controls

$y^{t, x}(s) \neq 0$ for all $s \in[t, T]$ if and only if $x \neq 0$. Now, if the initial state $x=0$, then $\|\hat{u}(s)\| \leq$ $k\left\|y^{t, x}(s)\right\|=0, s \in[t, T]$. Clearly, $\hat{u}(s) \equiv 0$ is the open-loop control associated with the following feedback control in $u_{k}^{F}$ :

$$
\varphi(\cdot, \cdot)=0, \quad(s, y) \in[0, T] \times \mathbb{R}^{n} .
$$

If $x \neq 0$, we take the linear feedback control $\varphi(\cdot, \cdot)$ as follows:

$$
\varphi(s, y)=\chi_{[t, T]}(s) \frac{\hat{u}(s) y^{t, x}(s)^{\top}}{\left\langle y^{t, x}(s), y^{t, x}(s)\right\rangle} y, \quad(s, y) \in[0, T] \times \mathbb{R}^{n},
$$

which fulfills (1.13). By the fact that (1.8) under the above feedback control admits a unique solution, we see that $\varphi \in U_{k}^{F}$ with which $\hat{u}(\cdot)$ is the open-loop control associated. This proves our result.

Now we introduce an open-loop LQ problem with constraints as follows.

Problem $2.4(\mathrm{LQ})_{k}$. For any $(t, x) \in[0, T) \times \mathbb{R}^{n}$, find a $\bar{u}^{t, x}(\cdot) \in \mathcal{U}_{k}^{t, x}$ such that

$$
J\left(t, x ; \bar{u}^{t, x}(\cdot)\right)=\inf _{u(\cdot) \in \mathscr{U}_{k}^{t, x}} J(t, x ; u(\cdot)) \stackrel{\text { def }}{=} V^{k}(t, x) .
$$

We call $\bar{u}^{t, x}(\cdot)$ an optimal open-loop control of Problem 2.4 at $(t, x)$, and call $V^{k}(\cdot, \cdot)$ the open-loop value function of Problem 2.4. Note that since $u_{k}^{t, x}$ is not even convex, Problem 2.4 is not a standard optimal control problem. Since every feedback control $\varphi(\cdot, \cdot) \in U_{k}^{F}$ has the same cost as its associated open-loop control, it follows from Lemma 2.3 that for any $(t, x) \in[0, T] \times \mathbb{R}^{n}$,

$$
\begin{aligned}
V^{k}(t, x) & =\inf _{u(\cdot) \in \mathcal{U}_{k}^{t, x}} J(t, x ; u(\cdot))=\inf _{u(\cdot) \in \mathcal{U}_{k}^{F}(t, x)} J(t, x ; u(\cdot)) \\
& =\inf _{\varphi(\cdot, \cdot) \in \mathcal{U}_{k}^{F}} J(t, x ; \varphi(\cdot, \cdot)) \equiv \bar{V}^{k}(t, x),
\end{aligned}
$$

which indicates that Problem 1.6 has the same value as Problem 2.4. The following proposition gives a further relationship between Problems 1.6 and 2.4.

Proposition 2.5. (i) For any given initial condition $(t, x)$, if feedback control $\bar{\varphi}(\cdot, \cdot) \in U_{k}^{F}$ satisfies

$$
J(t, x ; \bar{\varphi})=\inf _{\varphi(\cdot, \cdot) \in \mathcal{U}_{k}^{F}} J(t, x ; \varphi)
$$

then the open-loop control $\bar{u}(\cdot)$ associated with $\bar{\varphi}(\cdot, \cdot)$ solves Problem 2.4 at $(t, x)$.

(ii) Let $(t, x) \in[0, T) \times \mathbb{R}^{n}$. If the open-loop control Problem 2.4 admits a solution at $(t, x)$, then there exists a $\bar{\varphi}(\cdot, \cdot) \in \mathcal{U}_{k}^{F}$ such that

$$
J(t, x ; \bar{\varphi}(\cdot, \cdot))=\inf _{\varphi(\cdot, \cdot) \in \mathcal{U}_{k}^{F}} J(t, x ; \varphi(\cdot, \cdot)) .
$$


Proof. (i) Suppose the $\bar{\varphi}(\cdot, \cdot) \in \mathcal{U}_{k}^{F}$ solves Problem 1.6 at $(t, x)$, and let $\bar{u}(\cdot)$ be the associated open-loop control. If $\bar{u}(\cdot)$ is not optimal for Problem 2.4 at $(t, x)$, then there exists a $\hat{u}(\cdot) \in u_{k}^{t, x}$ satisfying

$$
J(t, x ; \widehat{u}(\cdot))<J(t, x ; \bar{u}(\cdot)) .
$$

By Lemma 2.3, there exists a feedback control $\hat{\varphi}(\cdot, \cdot) \in \mathcal{U}_{k}^{F}$ such that its associated openloop control is $\hat{u}(\cdot)$. But then

$$
J(t, x ; \hat{\varphi}(\cdot, \cdot))=J(t, x ; \hat{u}(\cdot))<J(t, x ; \bar{u}(\cdot))=J(t, x ; \bar{\varphi}(\cdot, \cdot)),
$$

which contradicts the optimality of $\bar{\varphi}(\cdot, \cdot)$.

(ii) Let $\bar{u}(\cdot)$ be a solution of Problem 2.4 at $(t, x)$. As in the proof of Lemma 2.3, we construct a feedback control $\bar{\varphi}(\cdot, \cdot)$ by $(2.10)$ or $(2.11)$, respectively, for the cases of $x=0$ and $x \neq 0$, respectively. Since the open-loop control $\bar{u}(\cdot)$ has the same cost as the feedback $\operatorname{control} \bar{\varphi}(\cdot, \cdot)$, the following equation follows from $(2.13)$ :

$$
\bar{V}^{k}(t, x)=J(t, x ; \bar{\varphi}(\cdot, \cdot))=J(t, x ; \bar{u}(\cdot))=V^{k}(t, x) .
$$

This shows that (2.15) holds.

Note that since in general $u_{k}^{t, x}$ is strictly smaller than $L^{2}\left(t, T ; \mathbb{R}^{m}\right)$, one has

$$
V(t, x) \equiv \inf _{u(\cdot) \in L^{2}\left(t, T ; \mathbb{R}^{m}\right)} J(t, x, u(\cdot)) \leq V^{k}(t, x),
$$

and strict inequality could hold. We know that $x \mapsto V(t, x)$ is quadratic. The following example shows that the value function $V^{k}(t, x)$ could be nonquadratic in the state $x$, which implies that the strict inequality in the above must hold somewhere.

Example 2.6. Let $n=2, m=1, A=0, B=(1,1)^{\top}, Q=R=W=I_{2}$, and $k=1$, where $I_{2}$ is the $(2 \times 2)$ unit matrix. The value function $V^{k}(\cdot, \cdot)$ satisfies the following equation (see Section 3 for an explanation):

$$
\begin{gathered}
V_{t}(t, x, y)+2\left(x^{2}+y^{2}\right)-\left|V_{x}(t, x, y)+V_{y}(t, x, y)\right| \\
\cdot \sqrt{x^{2}+y^{2}}=0, \quad \text { if }\left|V_{x}(t, x, y)+V_{y}(t, x, y)\right| \geq 2 \sqrt{x^{2}+y^{2}}, \\
V_{t}(t, x, y)+\left(x^{2}+y^{2}\right)-\frac{1}{4}\left[V_{x}(t, x, y)+V_{y}(t, x, y)\right]^{2} \\
=0, \quad \text { if }\left|V_{x}(t, x, y)+V_{y}(t, x, y)\right|<2 \sqrt{x^{2}+y^{2}}, \\
V(T, x)=x^{2}+y^{2} .
\end{gathered}
$$

Suppose that there exists a symmetric matrix-valued function $\widehat{P}(\cdot)=\left(\begin{array}{ll}p_{1}(t) & p_{2}(t) \\ p_{2}(t) & p_{3}(t)\end{array}\right) \in C([0$, $T] ; \mathbb{R}^{2 \times 2}$ ), such that

$$
V^{k}(t, x, y)=\left\langle\left(\begin{array}{ll}
p_{1}(t) & p_{2}(t) \\
p_{2}(t) & p_{3}(t)
\end{array}\right)\left(\begin{array}{l}
x \\
y
\end{array}\right),\left(\begin{array}{l}
x \\
y
\end{array}\right)\right\rangle .
$$


10 Existence and uniqueness of globally optimal feedback controls

Then $\hat{P}(T)=I_{2}$, and by the continuity of $\hat{P}(\cdot)$ there exists an interval $\left[T_{0}, T\right]$ such that

$$
\|\hat{P}(t)-\hat{P}(T)\| \leq \varepsilon, \quad t \in\left[T_{0}, T\right]
$$

for some given $0<\varepsilon<1-\sqrt{2} / 2$. Let $\xi=(1,1)$. Take a neighborhood of $\xi$,

$$
\Omega_{\delta}(\xi) \equiv\{(x, y) \mid\|(x, y)-\xi\|<\delta\}
$$

with $\delta>0$ small enough so that any point $(x, y) \in \Omega_{\delta}(\xi)$ satisfies

$$
\begin{aligned}
& \left\|\frac{1}{2}\left[V_{x}^{k}(t, x, y)+V_{y}^{k}(t, x, y)\right]\right\| \\
& =\left\|(1,1) \hat{P}(t)\left(\begin{array}{l}
x \\
y
\end{array}\right)\right\| \geq\left\|(1,1) \hat{P}(T)\left(\begin{array}{l}
x \\
y
\end{array}\right)\right\|-\sqrt{2}\|\hat{P}(t)-\hat{P}(T)\|\left\|\left(\begin{array}{l}
x \\
y
\end{array}\right)\right\| \\
& =|x+y|-\sqrt{2} \varepsilon \sqrt{x^{2}+y^{2}}>\sqrt{x^{2}+y^{2}} .
\end{aligned}
$$

Note that in the current case, (2.20) reads

$$
\begin{array}{r}
\left\langle\left(\begin{array}{ll}
\dot{p}_{1}(t) & \dot{p}_{2}(t) \\
\dot{p}_{2}(t) & \dot{p}_{3}(t)
\end{array}\right)\left(\begin{array}{l}
x \\
y
\end{array}\right),\left(\begin{array}{l}
x \\
y
\end{array}\right)\right\rangle+2\left(x^{2}+y^{2}\right) \\
=\left|p_{1}(t) x+p_{2}(t) x+p_{2}(t) y+p_{3}(t) y\right| \sqrt{x^{2}+y^{2}} .
\end{array}
$$

Clearly, for $(t, x, y) \in\left[T_{0}, T\right] \times \Omega_{\delta}(\xi)$, the left-hand side is quadratic in $(x, y)$, whereas the right-hand side is nonquadratic in $(x, y)$. This leads to a contradiction. Therefore, the value function $V^{k}(t, x, y)$ must not be quadratic in $(x, y)$.

By a direct calculation, it follows that

$$
V_{t}(T, \xi)=2>4(\sqrt{2}-1)=V_{t}^{k}(T, \xi)
$$

which, together with $V(T, \xi)=V^{k}(T, \xi)$, implies that

$$
V(t, \xi)<V^{k}(t, \xi)
$$

holds on some interval $[\widehat{T}, T]$, for $\xi$ in a bounded domain.

\section{Main results}

In this section, we first introduce a standard open-loop optimal control problem, via which we will construct a globally optimal closed-loop control which uniquely solves Problem 1.6. In what follows, we will assume condition (1.5).

By the discussion in Section 2, we know that Problem 2.4 has a very close connection with Problem 1.6. Hence, it is a hope that one can solve Problem 1.6 via solving Problem 2.4. But Problem 2.4 is not a standard open-loop optimal control problem. Consequently, many standard results are not applicable. To overcome the difficulty, we will 
introduce a standard open-loop optimal control problem which is essentially equivalent to Problem 2.4. More precisely, we consider the following system:

$$
\begin{gathered}
\dot{y}(s)=A(s) y(s)+B(s) K(s) y(s), \quad s \in[t, T], \\
y(t)=x \in \mathbb{R}^{n},
\end{gathered}
$$

where $K(\cdot)$ is the control variable belonging to the admissible control set

$$
\mathscr{K}_{k} \stackrel{\text { def }}{=}\left\{K(\cdot) \in L^{2}\left(0, T ; \mathbb{R}^{m \times n}\right) \mid\|K(s)\| \leq k\right\} .
$$

The corresponding cost functional is defined by

$$
J(t, x ; K(\cdot))=\int_{t}^{T}[\langle Q(s) y(s), y(s)\rangle+\langle R(s) K(s) y(s), K(s) y(s)\rangle] d s+\langle M y(T), y(T)\rangle .
$$

We propose following problem.

Problem $3.1(\mathrm{LQ})_{k}^{L}$. For any given $(t, x) \in[0, T] \times \mathbb{R}^{n}$, find a $\bar{K}(\cdot) \in \mathcal{K}_{k}$ such that

$$
J(t, x ; \bar{K}(\cdot))=\inf _{K(\cdot) \in \mathscr{K}_{k}} J(t, x ; K(\cdot)) \equiv \hat{V}^{k}(t, x),
$$

where and hereafter the superscript " $L$ " means linearity.

Denote

$$
u_{k}^{L} \stackrel{\text { def }}{=}\left\{\varphi(s, y)=K(s) y, \forall(s, y) \in[0, T] \times \mathbb{R}^{n} \mid K(\cdot) \in \mathcal{K}_{k}\right\} \subseteq u_{k}^{F} .
$$

It is clear that Problem 3.1 is a linear feedback control problem. We call $\hat{V}^{k}(\cdot, \cdot)$ the value function of Problem 3.1. Note that Problem 3.1 is a standard (nonlinear) open-loop optimal control problem. By the classical optimal control theory, $\hat{V}^{k}(\cdot, \cdot)$ is the unique viscosity solution of the following HJB equation:

$$
\begin{gathered}
V_{t}(t, x)+\min _{\|K\| \leq k}\left\{\left\langle V_{x}(t, x), A(t) x+B(t) K x\right\rangle+\langle Q(t) x, x\rangle\right. \\
+\langle R(t) K x, K x\rangle\}=0, \quad(t, x) \in[0, T] \times \mathbb{R}^{n}, \\
V(T, x)=\langle M x, x\rangle .
\end{gathered}
$$

Note that feedback controls (2.10) and (2.11) belong to the above linear feedback control set $u_{k}^{L}$. In fact, we can show that $U_{k}^{t, x}$ is also an open-loop control set associated with $u_{k}^{L}$. Thus

$$
\widehat{V}^{k}(t, x)=\bar{V}^{k}(t, x)=V^{k}(t, x), \quad(t, x) \in[0, T] \times \mathbb{R}^{n} .
$$

Consequently, $\bar{V}^{k}(\cdot, \cdot)$ also satisfies the above HJB equation in the viscosity solution sense. Note that the value function $V(\cdot, \cdot)$ of Problem 1.1 satisfies the following HJB 
12 Existence and uniqueness of globally optimal feedback controls

equation in the classical sense (and therefore also in the sense of viscosity solutions):

$$
\begin{gathered}
V_{t}(t, x)+\inf _{u \in \mathbb{R}^{m}}\left\{\left\langle V_{x}(t, x), A(t) x+B(t) u\right\rangle+\langle Q(t) x, x\rangle\right. \\
+\langle R(t) u, u\rangle\}=0, \quad(t, x) \in[0, T] \times \mathbb{R}^{n}, \\
V(T, x)=\langle M x, x\rangle .
\end{gathered}
$$

We see that (3.6) and (3.8) are significantly different. As an example, (2.20) is the value function equation of Example 2.6. Furthermore, we have the following results similar to Proposition 2.5.

Proposition 3.2. (i) If $\bar{K}(\cdot)$ is a solution to Problem 3.1 at $(t, x)$ and $\bar{y}(\cdot)$ is the corresponding state trajectory of (3.1), then open-loop control

$$
\bar{u}(\cdot)=\bar{K}(\cdot) \bar{y}(\cdot)
$$

solves Problem 2.4 at $(t, x)$.

(ii) If for given $(t, x) \in[0, T] \times \mathbb{R}^{n}$, there exists a $\bar{u}^{t, x}(\cdot)$ solving Problem 2.4 at $(t, x)$ and $y^{t, x}(\cdot)$ is corresponding state trajectory of (1.1), then the control

$$
\bar{K}(\cdot)= \begin{cases}0, & \text { if } x=0, \\ \chi_{[t, T]}(\cdot) \frac{\bar{u}^{t, x}(\cdot) y^{t, x}(\cdot)^{\top}}{\left\langle y^{t, x}(\cdot), y^{t, x}(\cdot)\right\rangle}, & \text { if } x \neq 0,\end{cases}
$$

solves Problem 3.1 at $(t, x)$.

We emphasize that the significance of the above result is that the solution of Problem 2.4 can be derived by that of Problem 3.1 which is a standard open-loop optimal control problem.

Next, we present results on the existence and uniqueness of the solution to Problem 2.4 .

Theorem 3.3. Let (1.5) hold. Then for any $(t, x) \in[0, T] \times \mathbb{R}^{n}$, Problem 2.4 admits an open-loop optimal control at $(t, x)$.

Proof. By Proposition 3.2, it is clear that we need only to prove that there exists a $\bar{K}(\cdot) \in$ $\mathscr{K}_{k}$ which solves Problem 3.1 at $(t, x)$. Note that the set

$$
\begin{gathered}
\left\{\left(Z_{0}, Z\right) \in \mathbb{R} \times \mathbb{R}^{n} \mid Z_{0} \geq\langle y, Q(s) y\rangle+\langle K y, R(s) K y\rangle,\right. \\
Z=A(s) y+B(s) K y, \quad \text { for some }\|K\| \leq k\}
\end{gathered}
$$

is convex and closed for any $(s, y) \in[t, T] \times \mathbb{R}^{n}$. Thus, the so-called Cesari condition holds [5], which implies the existence of $\bar{K}(\cdot)$. Clearly, the open-loop control associated with $\bar{\varphi}(s, y)=\bar{K}(s) y$ solves Problem 2.4 at $(t, x)$ by Proposition 3.2.

To obtain the uniqueness of the optimal open-loop control for Problem 2.4, we need the following lemma. 
Lemma 3.4. Let (1.5) hold and let $Q(\cdot)$ be uniformly positive definite. Then there exists a $\delta>0$ such that the following two-point boundary value problem has at most one solution for any $(k, t, x) \in[0, \delta] \times[0, T] \times \mathbb{R}^{n}$ (s is suppressed below):

$$
\begin{gathered}
\dot{y}=A y-\min \left\{\frac{1}{2}\left\|B^{\top} \psi\right\|, k\|y\|\right\} \frac{B B^{\top} \psi}{\left\|B^{\top} \psi\right\|}, \\
\dot{\psi}=-A^{\top} \psi-2 Q y+\left[\frac{\left\|B^{\top} \psi\right\|}{\|y\|}-2 k\right]^{+} \min \left\{\frac{\left\|B^{\top} \psi\right\|}{2\|y\|}, k\right\} y, \\
y(t)=x, \quad \psi(T)=2 M y(T),
\end{gathered}
$$

where $[c]^{+}=\max \{c, 0\}$ and, as a convention, assume that

$$
\begin{gathered}
\min \left\{\frac{1}{2}\left\|B^{\top} \psi\right\|, k\|y\|\right\} \frac{B B^{\top} \psi}{\left\|B^{\top} \psi\right\|}=0, \quad \text { for } B^{\top} \psi=0, \\
{\left[\frac{\left\|B^{\top} \psi\right\|}{\|y\|}-2 k\right]^{+} \min \left\{\frac{\left\|B^{\top} \psi\right\|}{2\|y\|}, k\right\} y=0, \quad \text { for } y=0 .}
\end{gathered}
$$

Proof. Suppose that $(y(\cdot), \psi(\cdot))$ is a solution to (3.12). Then similar to the proof of Proposition 2.1, using the first equation in (3.12), for any $r, s \in[t, T]$,

$$
\|y(r)\| \leq C\|y(s)\|+C k \int_{s}^{r}\|B(\tau) \mid\|\|y(\tau)\| d \tau .
$$

Hence, by Gronwall's inequality, we have

$$
\|y(r)\| \leq C\|y(s)\|, \quad \forall r, s \in[t, T] .
$$

Next, from the second equation in (3.12) and the terminal condition, we have

$$
\|\psi(s)\| \leq C\|y(T)\|+C \int_{s}^{T}(k\|\psi(\tau)\|+\|y(\tau)\|) d \tau .
$$

By Gronwall's inequality, together with (3.15), we have

$$
\begin{aligned}
\|\psi(s)\| & \leq C\|y(T)\|+C \int_{s}^{T}\|y(\tau)\| d \tau \\
& \leq C\|y(s)\|+C \int_{s}^{T}\|y(s)\| d \tau \leq C\|y(s)\|,
\end{aligned}
$$

where $C$ is independent of $(k, t, x) \in\left[0, K_{0}\right] \times[0, T] \times \mathbb{R}^{n}\left(K_{0}\right.$ is a fixed positive constant).

Now, suppose that $\left(y_{1}(\cdot), \psi_{1}(\cdot)\right)$ and $\left(y_{2}(\cdot), \psi_{2}(\cdot)\right)$ are two solutions of (3.12) for the fixed $(t, x)$. Therefore (3.16) and (3.17) hold for $\left(y_{i}(\cdot), \psi_{i}(\cdot)\right), i=1,2$. Let

$$
(\bar{y}(\cdot), \bar{\psi}(\cdot))=\left(y_{1}(\cdot)-y_{2}(\cdot), \psi_{1}(\cdot)-\psi_{2}(\cdot)\right) .
$$

Then

$$
\bar{y}(t)=0, \quad \bar{\psi}(T)=2 M \bar{y}(T) .
$$


14 Existence and uniqueness of globally optimal feedback controls

To prove the uniqueness of (3.12), we need the following estimate. First, it follows from (3.12) and (3.16) that

$$
\begin{aligned}
&\langle\bar{y}, d \bar{\psi} / d s\rangle+\left\langle\bar{y}, A^{\top} \bar{\psi}\right\rangle+2\langle\bar{y}, Q \bar{y}\rangle \\
&=\left\langle\bar{y},\left[\frac{\left\|B^{\top} \psi_{2}\right\|}{\left\|y_{2}\right\|}-2 k\right]^{+} \min \left\{\frac{\left\|B^{\top} \psi_{2}\right\|}{2\left\|y_{2}\right\|}, k\right\} \bar{y}\right\rangle \\
&+\left\langle\bar{y},\left[\frac{\left\|B^{\top} \psi_{1}\right\|}{\left\|y_{1}\right\|}-2 k\right]^{+} \min \left\{\frac{\left\|B^{\top} \psi_{1}\right\|}{2\left\|y_{1}\right\|}, k\right\} y_{1}\right\rangle \\
&-\left\langle\bar{y},\left[\frac{\left\|B^{\top} \psi_{2}\right\|}{\left\|y_{2}\right\|}-2 k\right]^{+} \min \left\{\frac{\left\|B^{\top} \psi_{2}\right\|}{2\left\|y_{2}\right\|}, k\right\} y_{1}\right\rangle \\
& \leq k C\|\bar{y}\|^{2}+k\|\bar{y}\|\left\|y_{1}\right\|\left|\left[\frac{\left\|B^{\top} \psi_{1}\right\|}{\left\|y_{1}\right\|}-2 k\right]^{+}-\left[\frac{\left\|B^{\top} \psi_{2}\right\|}{\left\|y_{2}\right\|}-2 k\right]^{+}\right| \\
& \leq k C\|\bar{y}\|^{2}+k\|\bar{y}\|\left\|y_{1}\right\|\left|\frac{\left\|B^{\top} \psi_{1}\right\|}{\left\|y_{1}\right\|}-\frac{\left\|B^{\top} \psi_{2}\right\|}{\left\|y_{2}\right\|}\right| \\
& \leq k C\|\bar{y}\|^{2}+k\|\bar{y}\|\|B\|\left[\left\|\psi_{1}-\psi_{2}\right\|+\frac{\left\|\psi_{2}\right\|}{\left\|y_{2}\right\|}\left\|y_{1}-y_{2}\right\|\right] \leq k C\|\bar{y}\|(\|\bar{y}\|+\|\bar{\psi}\|) .
\end{aligned}
$$

Next,

$$
\begin{aligned}
\left\langle\frac{d \bar{y}}{d s}, \bar{\psi}\right\rangle & -\langle A \bar{y}, \bar{\psi}\rangle \\
= & -\min \left\{\frac{1}{2}\left\|B^{\top} \psi_{1}\right\|, k\left\|y_{1}\right\|\right\}\left\langle\left(\frac{B^{\top} \psi_{1}}{\left\|B^{\top} \psi_{1}\right\|}-\frac{B^{\top} \psi_{2}}{\left\|B^{\top} \psi_{2}\right\|}\right), B^{\top} \bar{\psi}\right\rangle \\
& -\left\langle\left[\min \left\{\frac{1}{2}\left\|B^{\top} \psi_{1}\right\|, k\left\|y_{1}\right\|\right\}-\min \left\{\frac{1}{2}\left\|B^{\top} \psi_{2}\right\|, k\left\|y_{2}\right\|\right\}\right] \frac{B B^{\top} \psi_{2}}{\left\|B^{\top} \psi_{2}\right\|}, \bar{\psi}\right\rangle \\
\leq & 0+\left|k\left\|y_{1}\right\|-k\left\|y_{2}\right\|\right|\left|\left\langle\frac{B B^{\top} \psi_{2}}{\left\|B^{\top} \psi_{2}\right\|}, \bar{\psi}\right\rangle\right| \leq k\|\bar{y}\|\|B\|\|\bar{\psi}\| \leq k C\|\bar{y}\|\|\bar{\psi}\| .
\end{aligned}
$$

Finally, we can obtain by a similar method as (3.20)

$$
\begin{aligned}
\frac{1}{2} \frac{d\left(\|\bar{\psi}\|^{2}\right)}{d s}=\langle\bar{\psi}, \bar{\psi} / d s\rangle & \geq-\left\langle\bar{\psi}, A^{\top} \bar{\psi}\right\rangle-2\langle\bar{\psi}, Q \bar{y}\rangle-k C\|\bar{\psi}\|(\|\bar{y}\|+\|\bar{\psi}\|) \\
& \geq-C\left(\|\bar{\psi}\|^{2}+\|\bar{\psi} \mid\| \bar{y} \|\right) \geq-C\|\bar{\psi}\|^{2}-C\|\bar{y}\|^{2},
\end{aligned}
$$

which leads to

$$
\frac{d\left(e^{2 C(s-T)}\|\bar{\psi}\|^{2}\right)}{d s} \geq-2 C e^{2 C(s-T)}\|\bar{y}(s)\|^{2} .
$$


Hence, one has

$$
\int_{s}^{T}\|\bar{\psi}(\tau)\|^{2} d \tau \leq C\left[\|\bar{\psi}(T)\|^{2}+\int_{s}^{T}\|\bar{y}(\tau)\|^{2} d \tau\right] .
$$

Now we show the uniqueness of the solution to (3.12). Since $Q(\cdot)$ is uniformly positive definite on $[0, T]$, one has

$$
q \triangleq \min _{t \in[0, T]} \min _{\eta \neq 0}\left\{\frac{\langle\eta, Q(t) \eta\rangle}{\langle\eta, \eta\rangle}\right\}>0 .
$$

It follows from (3.19), (3.20), (3.21), (3.24), and (3.25) that

$$
\begin{aligned}
\langle\bar{y}(T), M \bar{y}(T)\rangle & =\langle\bar{y}(T), \bar{\psi}(T)\rangle-\langle\bar{y}(t), \bar{\psi}(t)\rangle \\
& =\int_{t}^{T}\left\langle\bar{y}(s), \frac{d \bar{\psi}(s)}{d s}\right\rangle+\left\langle\frac{d \bar{y}(s)}{d s}, \bar{\psi}(s)\right\rangle d s \\
& \leq \int_{t}^{T}-2\langle\bar{y}(s), Q(s) \bar{y}(s)\rangle+k C\|\bar{y}(s)\|(\|\bar{y}(s)\|+\|\bar{\psi}(s)\|) d s \\
& \leq \int_{t}^{T}\left[-2 q\|\bar{y}(s)\|^{2}+k C\|\bar{y}(s)\|^{2}+k C\|\bar{\psi}(s)\|^{2}\right] d s \\
& \leq \int_{t}^{T}\left[-2 q\|\bar{y}(s)\|^{2}+k C\|\bar{y}(s)\|^{2}\right] d s+k C\|\bar{\psi}(T)\|^{2} \\
& =\int_{t}^{T}-(2 q-k C)\|\bar{y}(s)\|^{2} d s+k C\langle M \bar{y}(T), M \bar{y}(T)\rangle \\
& \leq \int_{t}^{T}-(2 q-k C)\|\bar{y}(s)\|^{2} d s+k C\langle\bar{y}(T), M \bar{y}(T)\rangle,
\end{aligned}
$$

where the last inequality holds because

$$
\langle\widehat{M} x, \widehat{M} x\rangle \leq\|\widehat{M}\|\langle x, \widehat{M} x\rangle
$$

for any positive semidefinite matrix $\widehat{M} \in \mathbb{R}^{n \times n}$ and $x \in \mathbb{R}^{n}$. Thus

$$
(1-k C)\langle\bar{y}(T), M \bar{y}(T)\rangle \leq \int_{t}^{T}-(2 q-k C)\|\bar{y}(s)\|^{2} d s .
$$

Let $\delta \triangleq \min \{1 / C, 2 q / C\}$. If $k<\delta$, the above implies

$$
\bar{y}(\cdot) \equiv 0
$$

which, together with (3.19) and (3.24), leads to

$$
\bar{\psi}(\cdot) \equiv 0 .
$$

Therefore the two-point boundary value problem (3.11) admits at most one solution. 
Remark 3.5. A rough estimate shows that a lower bound of $\delta$ will be $1 / C_{3}$, where $C_{3}$ is defined as follows: if

$$
\begin{gathered}
a=\sup _{t \in[0, T]}\|A(t)\|, \quad b=\sup _{t \in[0, T]}\|B(t)\|, \\
k_{0}=\sup _{t \in[0, T]}\left\|B^{\top}(t) P(t)\right\|, \quad q=\sup _{t \in[0, T]}\|Q(t)\|, \\
C_{1}=\max \{1,2 q, b\} \exp (a t), \quad C_{2}=C_{1} T \exp \left(k_{0} C_{1} T+a T+b T\right),
\end{gathered}
$$

then

$$
C_{3}=2 C_{2} T \exp \left(2 C_{2} T\right)
$$

Besides, the condition that $Q(\cdot)$ is uniformly positive definite can be replaced by another condition that matrix $M$ is positive definite, to ensure the uniqueness of the solution for the two-point boundary value problem (3.12). Here we omit the proof.

Now we present the uniqueness of the solution for Problem 2.4.

Theorem 3.6. Let (1.5) hold and let $Q(\cdot)$ be uniformly positive definite. Then there exists a $\delta>0$ such that for any given $(k, t, x) \in[0, \delta] \times[0, T) \times \mathbb{R}^{n}$, Problem 2.4 admits at most one solution at $(t, x)$.

Proof. Without loss of generality, we assume that $R=I$. Clearly, control $u(\cdot)=0$ is the unique admissible open-loop control of Problem 2.4 at $(0,0)$. Thus it is also optimal. Now we fix $x \neq 0$.

Suppose that $y_{1}(\cdot), y_{2}(\cdot)$ are corresponding state trajectories, respectively, for optimal open-loop control $u_{1}(\cdot), u_{2}(\cdot)$ of Problem 2.4 at $(t, x)$. Then $y_{1}(s) \neq 0, y_{2}(s) \neq 0$ for any $s \in[t, T]$ by Proposition 2.1. As shown in Proposition 3.2,

$$
\bar{K}_{i}(\cdot)=\frac{u_{i}(\cdot) y_{i}^{\top}(\cdot)}{\left\|y_{i}(\cdot)\right\|^{2}}, \quad i=1,2
$$

solve Problem 3.1 at $(t, x)$. By Pontraygin's maximum principle, there exist adjoint functions $\psi_{1}(\cdot)$ and $\psi_{2}(\cdot)$ such that

$$
\begin{gathered}
\dot{y}_{i}=A y_{i}+B \bar{K}_{i} y_{i}, \\
\dot{\psi}_{i}=-\left(A+B \bar{K}_{i}\right)^{\top} \psi_{i}-2 Q y_{i}-2 \bar{K}_{i}^{\top} \bar{K}_{i} y_{i}, \\
y_{i}(t)=x, \psi_{i}(T)=2 M y_{i}(T), \\
\left\langle\psi_{i}, A y_{i}+B \bar{K}_{i} y_{i}\right\rangle+\left\langle\bar{K}_{i} y_{i}, \bar{K}_{i} y_{i}\right\rangle \leq\left\langle\psi_{i}, A y_{i}+B K y_{i}\right\rangle+\left\langle K y_{i}, K y_{i}\right\rangle
\end{gathered}
$$

hold for any $K \in \mathbb{R}^{m \times n},\|K\| \leq k$. But it is a pity that $\bar{K}$ cannot be obtained directly by the above. Note that $u_{i}=\bar{K}_{i} y_{i}$ solves the following problem:

$$
\min _{\|u\| \leq k\left\|y_{i}\right\|}\left\{\left\langle\psi_{i}, A y_{i}+B u\right\rangle+\langle u, u\rangle\right\}
$$


respectively, for $i=1,2$. Geometrically, $u_{i}$ is nothing but the projection of the vector $\zeta_{i}=$ $-(1 / 2) B^{\top} \psi_{i}$ onto the convex set $\Omega_{i}=\left\{\xi \in \mathbb{R}^{m} \mid\|\xi\| \leq k\left\|y_{i}\right\|\right\}$. Thus we denote

$$
u_{i} \equiv P_{\Omega_{i}}\left(\zeta_{i}\right)=-\min \left\{\frac{1}{2}\left\|B^{\top} \psi_{i}\right\|, k\left\|y_{i}\right\|\right\} \frac{B^{\top} \psi_{i}}{\left\|B^{\top} \psi_{i}\right\|},
$$

where $P_{\Omega_{i}}$ is the projection onto $\Omega_{i}$. Then the representation of $\bar{K}_{i}$ is derived by (3.33):

$$
\bar{K}_{i}=-\frac{\min \left\{(1 / 2)\left\|B^{\top} \psi_{i}\right\|, k\left\|y_{i}\right\|\right\} B^{\top} \psi_{i} y_{i}^{\top}}{\left\|B^{\top} \psi_{i}\right\|\left\|y_{i}\right\|^{2}} .
$$

Substitute the above equation into (3.34). We have that both $\left(y_{1}, \psi_{1}\right)$ and $\left(y_{2}, \psi_{2}\right)$ can solve the two-point boundary value problem (3.12). By Lemma 3.4, $\left(y_{1}, \psi_{1}\right)=\left(y_{2}, \psi_{2}\right)$ holds if $k$ is small enough, which implies $u_{1}=u_{2}$ by (3.37). In a word, there exists a constant $\delta>0$ such that Problem 2.4 admits at most one optimal open-loop control at $(t, x)$ when $k<\delta$.

We emphasize that an optimal control has the following representation:

$$
\bar{u}(s)=-\min \left\{\frac{1}{2}\left\|B^{\top}(s) \psi(s)\right\|, k\|y(s)\|\right\} \frac{B^{\top}(s) \psi(s)}{\left\|B^{\top}(s) \psi(s)\right\|}, \quad s \in[t, T],
$$

by the proof of the above theorem as (3.37) if it exists, where $(y(\cdot), \psi(\cdot))$ is the corresponding state variable and adjoint variable. Further, the solution to Problem 2.4 at $(t, x)$ fulfills

$$
\bar{u}(\cdot) \in C\left([t, T] ; \mathbb{R}^{m}\right), \quad(t, x) \in[0, T) \times \mathbb{R}^{n},
$$

which follows from the continuity of $y(\cdot)$ and $\psi(\cdot)$.

We are now at the position to state and prove the main result of this section.

Theorem 3.7. Let (1.5) hold and let $Q(\cdot)$ be uniformly positive definite. Then there exists a $\delta>0$ such that Problem 1.6 admits a unique globally optimal continuous feedback control for any $k \leq \delta$.

Proof. By Lemma 3.4 and Theorem 3.6, Problem 2.4 admits a unique solution at $(t, x)$ if $k$ is small enough for any given $(t, x) \in[0, T] \times \mathbb{R}^{n}$. We denote $\bar{u}^{t, x}(\cdot)$ to be the solution to Problem 2.4 at $(t, x)$, and $y^{t, x}(\cdot)$ to be the corresponding optimal trajectory. Construct a feedback control $\bar{\varphi}:[0, T) \times \mathbb{R}^{n} \rightarrow \mathbb{R}^{m}$ by the synthesis method as follows:

$$
\bar{\varphi}(t, x)=\bar{u}^{t, x}(t), \quad(t, x) \in[0, T] \times \mathbb{R}^{n}
$$

which is well defined by the continuity of $\bar{u}^{t, x}(\cdot)$. Further, (1.14) holds by the definition of control set $u_{k}^{t, x}$.

We prove that $\bar{\varphi}$ solves Problem 1.6 by three steps. First, we prove the continuity of $\bar{\varphi}(\cdot, \cdot)$. As shown in the proof of Theorem 3.6, there exists a $\psi^{t, x}$ which together with the state variable $y^{t, x}$, satisfies (3.12). On the other hand, Lemma 3.4 indicates that (3.12) admits at most one solution. Therefore, adjoint variable $\psi^{t, x}$ is unique and determined 
18 Existence and uniqueness of globally optimal feedback controls

by the initial condition $(t, x)$. Thus, function $\psi:[0, T] \times \mathbb{R}^{n} \rightarrow \mathbb{R}^{n}$,

$$
\psi(t, x)=\psi^{t, x}(t), \quad(t, x) \in[0, T] \times \mathbb{R}^{n},
$$

is also well defined. By the classical result on the continuous dependence of the solution with respect to the parameter, we obtain that the function $\psi(\cdot, \cdot)$ is continuous.

Let $\Omega(x)=\left\{\xi \in \mathbb{R}^{m} \mid\|\xi\| \leq k\|x\|\right\}$. Thus

$$
\left\|P_{\Omega(x)}(\xi)-P_{\Omega(y)}(\xi)\right\| \leq|k\|x\|-k\|y\||
$$

is valid for any $\xi \in \mathbb{R}^{n}$ since $\Omega(x)$ and $\Omega(y)$ are concentric spheres. Further

$$
\left\|P_{\Omega_{(x)}}(\xi)-P_{\Omega_{(x)}}(\eta)\right\| \leq\|\xi-\eta\|, \quad \xi, \eta \in \mathbb{R}^{n}
$$

holds by properties of the convex set [2]. It follows by the above two equations and (3.37) that

$$
\begin{aligned}
\left\|\bar{\varphi}(t, x)-\bar{\varphi}\left(t_{0}, x_{0}\right)\right\|= & \left\|\bar{u}^{t, x}(t)-\bar{u}^{t_{0}, x_{0}}\left(t_{0}\right)\right\| \\
= & \left\|P_{\Omega(x)}\left(-\frac{1}{2} B^{\top}(t) \psi^{t, x}(t)\right)-P_{\Omega(y)}\left(-\frac{1}{2} B^{\top}\left(t_{0}\right) \psi^{t_{0}, x_{0}}\left(t_{0}\right)\right)\right\| \\
\leq & \left\|P_{\Omega(x)}\left(-\frac{1}{2} B^{\top}(t) \psi^{t, x}(t)\right)-P_{\Omega(x)}\left(-\frac{1}{2} B^{\top}\left(t_{0}\right) \psi^{t_{0}, x_{0}}\left(t_{0}\right)\right)\right\| \\
& +\left\|P_{\Omega(x)}\left(-\frac{1}{2} B^{\top}\left(t_{0}\right) \psi^{t_{0}, x_{0}}\left(t_{0}\right)\right)-P_{\Omega(y)}\left(-\frac{1}{2} B^{\top}\left(t_{0}\right) \psi^{t_{0}, x_{0}}\left(t_{0}\right)\right)\right\| \\
\leq & \left\|-\frac{1}{2} B^{\top}(t) \psi^{t, x}(t)+\frac{1}{2} B^{\top}\left(t_{0}\right) \psi^{t_{0}, x_{0}}\left(t_{0}\right)\right\|+\left|k\|x\|-k\left\|x_{0}\right\|\right| \\
= & \left\|-\frac{1}{2} B^{\top}(t) \psi(t, x)+\frac{1}{2} B^{\top}\left(t_{0}\right) \psi\left(t_{0}, x_{0}\right)\right\|+\left|k\|x\|-k\left\|x_{0}\right\|\right| .
\end{aligned}
$$

Thus function $\bar{\varphi}(t, \cdot)$ is continuous due to the continuity of $\psi(\cdot, \cdot)$ and $B(\cdot)$.

Second, we prove the uniqueness of solution to the state equation under control $\bar{\varphi}(\cdot, \cdot)$ for any initial condition. To this aim, let $(t, x)$ be given. Then the adjoint variable $\psi^{t, x}(t)$ satisfies

$$
\begin{aligned}
\psi(t, x) & =\psi^{t, x}(t) \in D_{x}^{1,+} V^{k}(t, x) \\
& \equiv\left\{p \in \mathbb{R}^{n} \mid \varlimsup_{y \rightarrow x} \frac{V^{k}(t, y)-V^{k}(t, x)-\langle p, y-x\rangle}{\|y-x\|} \leq 0\right\},
\end{aligned}
$$

by the relationship between the adjoint variable and the value function for the Problem 3.1 (see [19]). Thus, the following equation holds:

$$
V^{k}(t, z) \leq V^{k}(t, y)+\int_{0}^{1}\langle\psi(t, y+\tau(z-y)), z-y\rangle d \tau
$$


for any $z, y \in \mathbb{R}^{n}$. Now take $z=x$ and let $y \rightarrow x$. It follows from the continuity of function $\psi(\cdot, \cdot)$ that

$$
\begin{aligned}
0 & \leq \varliminf_{y \rightarrow x} \frac{V^{k}(t, y)-V^{k}(t, x)-\int_{0}^{1}\langle\psi(t, x+\tau(x-y)), y-x\rangle d \tau}{\|y-x\|} \\
& =\lim _{y \rightarrow x} \frac{V^{k}(t, y)-V^{k}(t, x)-\langle\psi(t, x), y-x\rangle}{\|y-x\|},
\end{aligned}
$$

which, together with $\psi(t, x)=\psi^{t, x}(t) \in D_{x}^{1,+} V^{k}(t, x)$, implies that $V_{x}^{k}(\cdot, \cdot)$ exists and

$$
V_{x}^{k}(t, x)=\psi(t, x), \quad(t, x) \in[0, T] \times \mathbb{R}^{n} .
$$

Further, $V_{x}^{k}(\cdot, \cdot)$ is continuous because of the continuity of function $\psi(\cdot, \cdot)$.

On the other hand, it follows by the continuity of $\psi(\cdot, \cdot)$ and $\varphi(\cdot, \cdot)$ that

$$
\begin{aligned}
& \frac{1}{s-t}\left[V^{k}(s, x)-V^{k}(t, x)\right] \\
&=\frac{1}{s-t}\left[V^{k}\left(s, y^{t, x}(s)\right)-V^{k}(t, x)\right]-\frac{1}{s-t}\left[V^{k}\left(s, y^{t, x}(s)\right)-V^{k}(s, x)\right] \\
&=\frac{1}{s-t}\left[J\left(s, y^{t, x}(s) ; \bar{u}^{t, x}(\cdot)\right)-J\left(t, x ; \bar{u}^{t, x}(\cdot)\right)\right] \\
&-\frac{1}{s-t} \int_{t}^{s}\left\langle V_{x}^{k}\left(s, y^{t, x}(\tau)\right), A(\tau) y^{t, x}(\tau)+B(\tau) \bar{u}^{t, x}(\tau)\right\rangle d \tau \\
&=-\langle x, Q(t) x\rangle-\left\langle\bar{u}^{t, x}(t), R(t) \bar{u}^{t, x}(t)\right\rangle+o(s-t) \\
&-\frac{1}{s-t} \int_{t}^{s}\left\langle\psi\left(s, y^{t, x}(\tau)\right), A(\tau) y^{t, x}(\tau)+B(\tau) \bar{\varphi}\left(\tau, y^{t, x}(\tau)\right)\right\rangle d \tau \\
&=-\langle x, Q(t) x\rangle-\langle\bar{\varphi}(t, x), R(t) \bar{\varphi}(t, x)\rangle-\langle\psi(t, x), A(t) x+B(t) \bar{\varphi}(t, x)\rangle+o(s-t) \\
&=-\langle x, Q(t) x\rangle-\langle\bar{\varphi}(t, x), R(t) \bar{\varphi}(t, x)\rangle-\left\langle V_{x}^{k}(t, x), A(t) x+B(t) \bar{\varphi}(t, x)\right\rangle+o(s-t) .
\end{aligned}
$$

Thus $V_{t}^{k}(t, x)$ exists and

$$
\begin{aligned}
V_{t}^{k}(t, x) & +\left\langle V_{x}^{k}(t, x), A(t) x+B(t) \bar{\varphi}(t, x)\right\rangle \\
= & -\langle x, Q(t) x\rangle+\langle\bar{\varphi}(t, x), R(t) \bar{\varphi}(t, x)\rangle, \quad(t, x) \in[0, T] \times \mathbb{R}^{n} .
\end{aligned}
$$

Furthermore, the continuity of $\bar{\varphi}(\cdot, \cdot)$ leads to that of $V_{t}^{k}(\cdot, \cdot)$. Therefore the regularity of value function holds.

Let us proceed to prove that the solution of (1.8) is unique under the feedback control $\bar{\varphi}(\cdot, \cdot)$. By Bellman's dynamic programming, $\left.\bar{u}^{t, x}(\cdot)\right|_{[s, T]}$ solves Problem 2.4 at $\left(s, y^{t, x}(s)\right)$, for all $t<s<T$, where $\left.\bar{u}^{t, x}(\cdot)\right|_{[s, T]}$ is a restriction of $\bar{u}^{t, x}(\cdot)$ on $[s, T]$. It follows from the uniqueness of the solution of Problem 2.4 at $\left(s, y^{t, x}(s)\right)$ that

$$
\bar{u}^{t, x}(s)=\bar{u}^{s, y^{t, x}(s)}(s)=\bar{\varphi}\left(s, y^{t, x}(s)\right) .
$$


Thus

$$
\begin{aligned}
\dot{y}^{t, x}(s)=A(s) y^{t, x}(s)+B(s) \bar{u}^{t, x}(s) & \\
= & A(s) y^{t, x}(s)+B(s) \bar{\varphi}\left(s, y^{t, x}(s)\right), \quad s \in[t, T], \\
y^{t, x}(t)=x &
\end{aligned}
$$

which shows that $y^{t, x}(\cdot)$ is a solution to $(1.8)$ under $\bar{\varphi}(\cdot, \cdot)$ for the given $(t, x)$. Suppose that $y_{1}(\cdot)$ is another solution, which is different from $y^{t, x}(\cdot)$. Denote $u_{1}(\cdot)=\bar{\varphi}\left(\cdot, y_{1}(\cdot)\right)$. Then the following equation holds by (3.51):

$$
\begin{aligned}
J(t, x ; & \left.u_{1}(\cdot)\right) \\
& =\int_{t}^{T}\left[\left\langle y_{1}(s), Q(s) y_{1}(s)\right\rangle+\left\langle u_{1}(s), R(s) u_{1}(s)\right\rangle\right] d s+\left\langle M y_{1}(T), y_{1}(T)\right\rangle \\
& =\int_{t}^{T}\left[\left\langle y_{1}(s), Q(s) y_{1}(s)\right\rangle+\left\langle\bar{\varphi}\left(s, y_{1}(s)\right), R(s) \bar{\varphi}\left(s, y_{1}(s)\right)\right\rangle\right] d s+\left\langle M y_{1}(T), y_{1}(T)\right\rangle \\
& =-\int_{t}^{T}\left[V_{t}^{k}\left(s, y_{1}(s)\right)+V_{x}^{k}\left(s, y_{1}(s)\right)\right] d s+\left\langle M y_{1}(T), y_{1}(T)\right\rangle \\
& =-\int_{t}^{T} \frac{d V^{k}\left(s, y_{1}(s)\right)}{d s} d s+V^{k}\left(T, y_{1}(T)\right) \\
& =V^{k}\left(t, y_{1}(t)\right)=V^{k}(t, x) .
\end{aligned}
$$

Thus $u_{1}(\cdot)=\bar{u}^{t, x}(\cdot)$ holds by the uniqueness of the solution to Problem 2.4 at $(t, x)$, which implies $y_{1}(\cdot)=y^{t, x}(\cdot)$. This indicates that (1.8) under feedback control $\bar{\varphi}(\cdot, \cdot)$ admits a unique solution. Therefore, the continuous feedback control $\bar{\varphi}(\cdot, \cdot)$ belongs to $u_{k}^{F}$.

Third. It follows by $(3.52)$ that $\bar{u}^{t, x}(\cdot)$ is the open-loop control associated with the feedback $\bar{\varphi}$ at $(t, x)$. Then,

$$
J(t, x ; \bar{\varphi})=J\left(t, x ; \bar{u}_{k}^{t, x}\right)=V^{k}(t, x)=\bar{V}^{k}(t, x) .
$$

Therefore this feedback control $\bar{\varphi}(\cdot, \cdot)$ solves Problem 1.6. By the uniqueness of solution for Problem 2.4 at $(t, x)$, the optimal feedback control is also unique. Thus the proof is completed.

In above theorem, the assumption on the "smallness of $k$ " is purely technical to ensure that the two-point boundary value problem (3.12) admits a unique solution, it is left open whether this is the case in general.

To conclude this paper, we indicate a process of solving Problem 1.6. For any $(t, x) \in$ $[0, T] \times \mathbb{R}^{n}$, one first solves the two-point boundary value problem(3.12) to obtain 
$\left(y^{t, x}(\cdot), \psi^{t, x}(\cdot)\right)$. Then define $u^{t, x}(\cdot)$ by (3.39). Finally, define set $\bar{\varphi}(\cdot, \cdot)$ by (3.41). This $\bar{\varphi}(\cdot, \cdot)$ will be the optimal closed-loop control of Problem 1.6.

\section{Acknowledgments}

The author would like to thank Professor H. Lou for many constructive suggestions. This work is partially supported by the National Natural Science Foundation of China under Grant 10371024.

\section{References}

[1] B. D. O. Anderson and J. B. Moore, Linear Optimal Control, Prentice-Hall, New Jersey, 1971.

[2] L. D. Berkovitz, Convexity and Optimization in $R^{n}$, Pure and Applied Mathematics (New York), John Wiley \& Sons, New York, 2002.

[3] P. Brunovský, Regular synthesis for the linear-quadratic optimal control problem with linear control constraints, Journal of Differential Equations 38 (1980), no. 3, 344-360.

[4] J. Casti, The linear-quadratic control problem: some recent results and outstanding problems, SIAM Review 22 (1980), no. 4, 459-485.

[5] L. Cesari, Existence of solutions and existence of optimal solutions, Mathematical Theories of Optimization (Genova, 1981), Lecture Notes in Math., vol. 979, Springer, Berlin, 1983, pp. 88-107.

[6] G. R. Chen, Closed-form solutions of a general inequality-constrained LQ optimal control problem, Applicable Analysis 41 (1991), no. 1-4, 257-279.

[7] Y. Z. Chen, Linear quadratic problems for time-varying systems: the cases without constraints and with the control energy constraint, Control Theory \& Applications 16 (1999), no. 4, 474-477.

[8] Z. Emirsajłow, Linear-quadratic control problem with terminal state constraints, Systems Analysis Modelling Simulation 4 (1987), no. 3, 227-240.

[9] __ A unified approach to optimal feedback in the infinite-dimensional linear-quadratic control problem with an inequality constraint on the trajectory or terminal state, IMA Journal of Mathematical Control and Information 8 (1991), no. 2, 179-208.

[10] H. Jaddu, Spectral method for constrained linear-quadratic optimal control, Mathematics and Computers in Simulation 58 (2002), no. 2, 159-169.

[11] R. Kalman, When is a linear control law optimal?, Transactions of the ASME Journal of Basic Engineering 86D (1964), 51-60.

[12] Y. Liu, S. Ito, H. W. J. Lee, and K. L. Teo, Semi-infinite programming approach to continuouslyconstrained linear-quadratic optimal control problems, Journal of Optimization Theory and Applications 108 (2001), no. 3, 617-632.

[13] D. H. Martin and D. H. Jacobson, Optimal control laws for a class of constrained linear-quadratic problems, Automatica 15 (1979), no. 4, 431-440.

[14] A. Matveev and V. Yakubovich, Nonconvex problems of global optimization: linear-quadratic control problems with quadratic constraints, Dynamics and Control 7 (1997), no. 2, 99-134.

[15] F. W. Sears, An Introduction to Thermodynamic: The Kinetic Theory of ASES, and Statistical Mechanics, Addison-Wesley, Massachusetts, 1956.

[16] G. Stefani and P. Zezza, Constrained regular LQ-control problems, SIAM Journal on Control and Optimization 35 (1997), no. 3, 876-900.

[17] D. D. Thompson and R. A. Volz, The linear quadratic cost problem with linear state constraints and the nonsymmetric Riccati equation, SIAM Journal on Control and Optimization 13 (1975), no. $1,110-145$.

[18] Y. Xu, Uniformly Lipschitz optimal feedback control problems, Dissertation, Fudan University, Shanghai, 2006. 
22 Existence and uniqueness of globally optimal feedback controls

[19] J. Yong and X. Y. Zhou, Stochastic Controls. Hamiltonian Systems and HJB Equations, Applications of Mathematics (New York), vol. 43, Springer, New York, 1999.

Yashan Xu: Institute of Mathematics, Fudan University, Shanghai 200433, China

E-mail address:021018046@fudan.edu.cn 


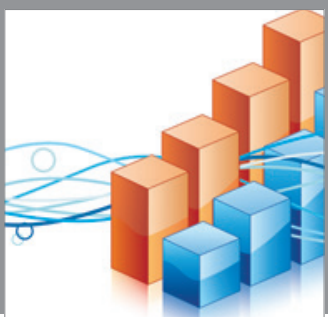

Advances in

Operations Research

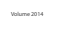

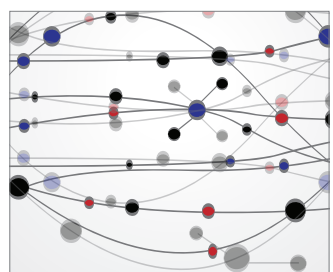

\section{The Scientific} World Journal
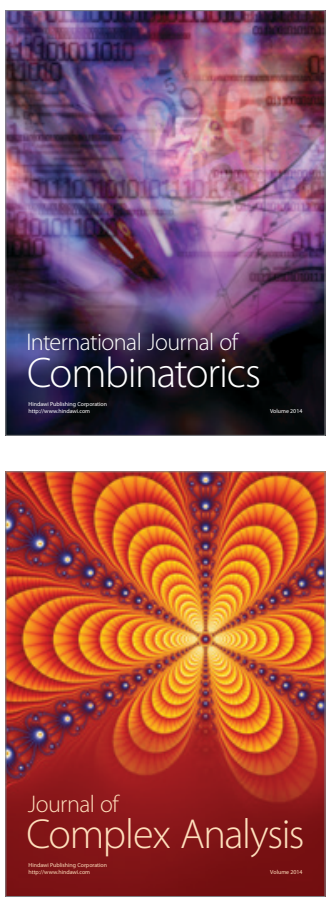

International Journal of

Mathematics and

Mathematical

Sciences
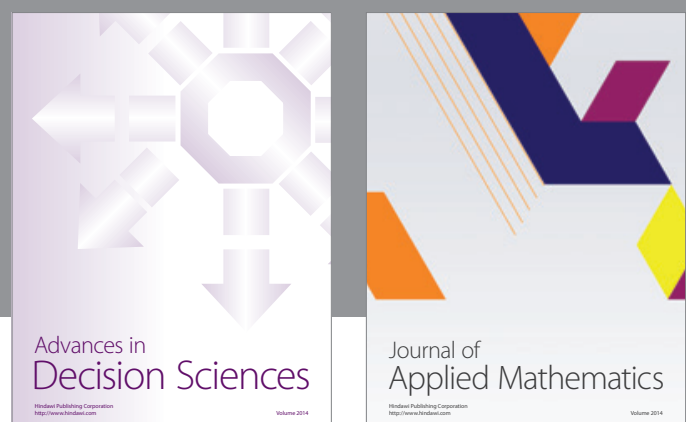

Journal of

Applied Mathematics
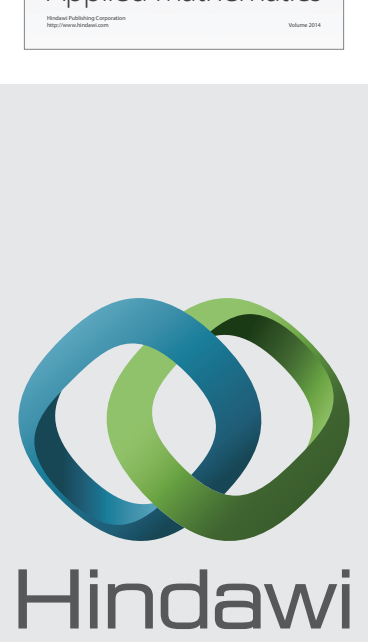

Submit your manuscripts at http://www.hindawi.com
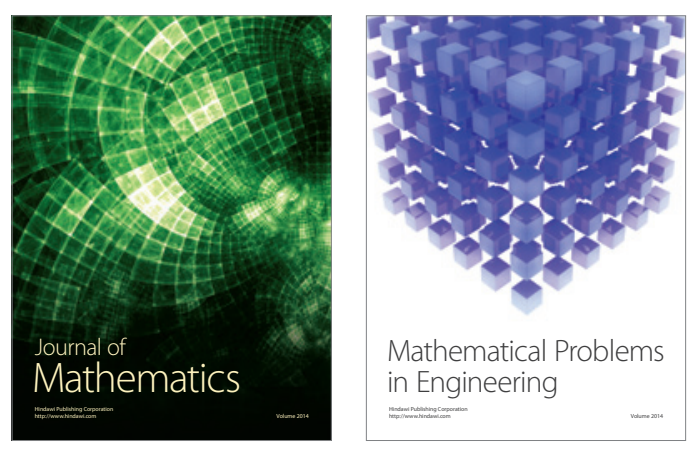

Mathematical Problems in Engineering
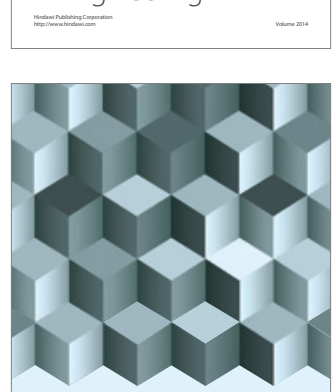

Journal of

Function Spaces
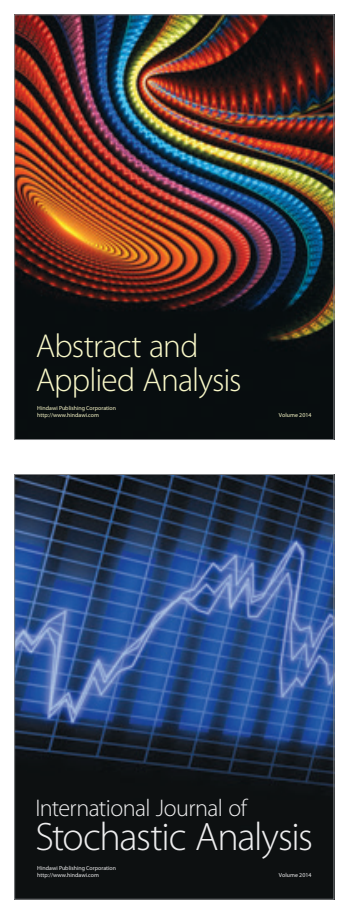

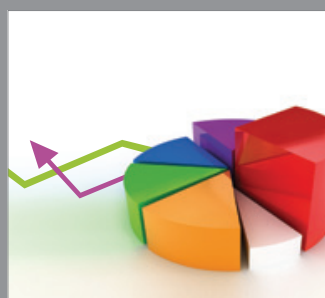

ournal of

Probability and Statistics

Promensencen
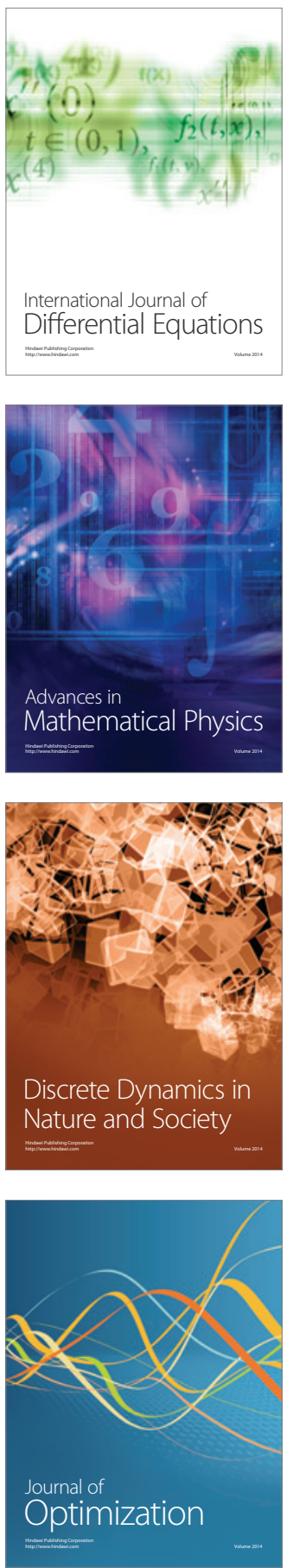